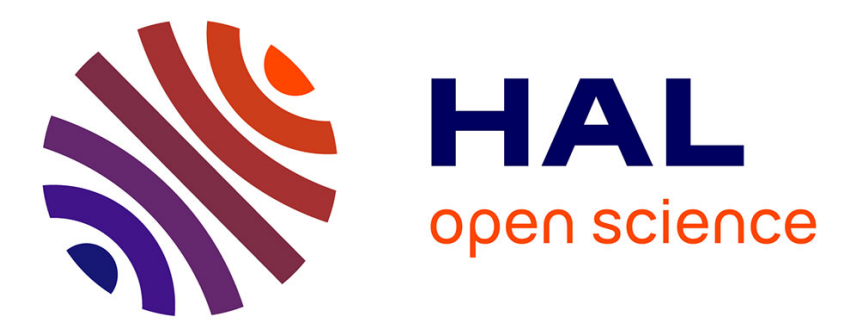

\title{
Compliant Joints Increase the Energy Efficiency of Bipedal Robot
}

\author{
Abdul Haq, Yannick Aoustin, Christine Chevallereau
}

\section{To cite this version:}

Abdul Haq, Yannick Aoustin, Christine Chevallereau. Compliant Joints Increase the Energy Efficiency of Bipedal Robot. The 14th International Conference on Climbing and Walking Robots and the Support Technologies for Mobile Machines (CLAWAR2011), Sep 2011, Paris, France. hal-00668813

\section{HAL Id: hal-00668813 https://hal.science/hal-00668813}

Submitted on 10 Feb 2012

HAL is a multi-disciplinary open access archive for the deposit and dissemination of scientific research documents, whether they are published or not. The documents may come from teaching and research institutions in France or abroad, or from public or private research centers.
L'archive ouverte pluridisciplinaire HAL, est destinée au dépôt et à la diffusion de documents scientifiques de niveau recherche, publiés ou non, émanant des établissements d'enseignement et de recherche français ou étrangers, des laboratoires publics ou privés. 


\title{
Compliant Joints Increase the Energy Efficiency of Bipedal Robot
}

\author{
A. Haq, Y. Aoustin and C. Chevallereau \\ LUNAM, Institut de Recherche en Communications et Cybernétique de Nantes \\ UMR CNRS 6597, CNRS, Université de Nantes, \\ 1 rue de la Noe, 44321 Nantes Cedex 3, France \\ E-mail: Abdul.Haq@irccyn.ec-nantes.fr, Yannick.Aoustin@irccyn.ec-nantes.fr \\ Christine.Chevallereau@irccyn.ec-nantes.fr
}

\begin{abstract}
The energetic effects of knee locking and addition of linear elastic members to different joints of a seven-link fully actuated planar bipedal robot were studied. The focus was on the reduction of energy consumption during walking. An impactless walking gait was studied and the energetic cost of walking was determined without joint stiffness and knee locking as a baseline for comparison. The gait trajectory was then optimized by adding spring to different joints, energetic cost of walk was then calculated at different walking speeds. Support knee was then mechanically locked and gait was optimized to find the cost of walking. The energetic cost of walking determined for the above two cases was then compared to the baseline cost. It was observed that addition of torsional springs at both knees can reduce the walking cost up to $62 \%$ at lower speeds and both hips up to $35 \%$ at high walking speeds with spring stiffness as an optimization parameter for both cases. Mechanically locking the support knee can reduce the cost of walking up to $84 \%$ at slow walking speeds with gait and knee locking angle optimized.
\end{abstract}

Keywords: biped, energetic efficiency, passive springs, compliance, impactless gait

\section{Motivation}

Energetic efficiency and stability are the fundamental criteria that can improve autonomy and task performance capabilities of humanoid robots. Researchers are trying to generate dynamically stable and energetically efficient bipedal gaits as close as possible to human walk. ${ }^{2,6,11}$ Studies shows that the legs of the humanoid robots consume more energy in the stance phase than in the swing phase. ${ }^{3}$ This difference in energy consumption is because of the demand of high torques to support the robot weight on the ground. Therefore, there is a space of energy optimization in the support 
leg.

The scope of this work is to investigate the energetic effects of knee locking and addition springs to different joints of a planar bipedal robot. The focus is on the reduction of energy consumption during walking.

\section{Introduction}

Recently, springs have been used to recover the lost energy, decrease the energy consumption and stabilize the walking gait. In most of the cases, springs are added to the ankle of the robot to store energy and use it when needed mainly during ankle push-off just before heel strike. Studies indicate that there is a direct trade-off between the toe-off impulse from the trailing leg and the rotational torque between the legs. ${ }^{5}$ Using the toeoff impulse alone to power gait is four times less energetically expensive then using the hip torque alone. ${ }^{4,5}$ Another method of reducing energy consumption is to mechanically lock the support knee and release the lock during swing phase. ${ }^{9}$ The knee-lock with active release mechanism is found to be technologically simple and energetically efficient. ${ }^{8}$ However, neither the combined effects of knee locking and addition of springs have been explored nor the effects of compliance on energy consumption have been presented in function of walking speed.

In this paper, we carried out four different studies, One on the robot with no springs and locking mechanism, second with spring in different articulations, third with knee locking during support phase and fourth by adding the spring to the joint which economize maximum energy and then locking the support knee in parallel with springs. We assumed that the support knee can be mechanically locked and the torque required is provided by the mechanical lock.

\section{Presentation of the Biped}

Our seven-link bipedal robot is a planer bipedal robot, composed of two identical legs and a torso. Each leg consists of a thigh, shin and a rigid flat foot. All joints are revolute, frictionless and can only move in the sagittal plane. Our biped is $2 \mathrm{D}$ equivalent of humanoid robot $\mathrm{HYDROiD}^{1}$ having body mass and length similar to those of a human.

The desired gait consists of single support phases separated by instantaneous impactless phase (the swing foot comes in contact with ground with zero velocity). Right and left feet are supposed to be the stance and swing foot respectively. The walking step starts with a single support phase and 
ends with impactless flat foot contact on the ground where the feet change their role i.e. the stance foot becomes swing foot and vice versa.

Following different types of studies were carried out on the biped:

case A. The robot trajectories were optimized and energetic cost of walk was calculated without adding springs or blocking the knee.

case B. Springs were added to the hip, knee or ankle joints of the robot.

case B1. Spring was added only to the support leg joints i.e. support ankle, knee or hip.

case B2. Identical springs were added to both joints of the ankle, knee or hip.

In all the cases where a spring was added to any of the joint, the spring constant $K$ was optimized along with the gait and the spring offset or bias angle $\theta_{0}$ was kept zero.

case C. Support knee was mechanically locked at transition between single support without adding springs at any of the joint. The knee remained locked during the entire support phase.

case C1. The knee locking angle $(\beta)$ was an optimization parameter for trajectory optimization.

case C2. Based on the numerical values obtained in case $\mathbf{C 1}$, a constant value of $\beta$ was selected and then the gait was optimized.

case D. Support knee was mechanically locked and identical springs were added to both hip joints. The knee locking angle $\beta$ and spring stiffness $K$ were optimized with gait trajectory.

\section{Model of the Biped and Optimization}

\subsection{Dynamic Model in Single Support}

The dynamic model can be developed by using the Lagrange formulation. In single support phase, the dynamic model, considering an implicit liaison of the stance foot with the ground, i.e. there is no take off and no slipping during the single support phase, can be written as in (1) for simple biped and in (2) for a biped with torsional springs.

$$
\mathbf{A}(\mathbf{q}) \ddot{\mathbf{q}}+\mathbf{C}(\mathbf{q}, \dot{\mathbf{q}}) \dot{\mathbf{q}}+\mathbf{G}(\mathbf{q})=\mathbf{B} \Gamma
$$

$$
\mathbf{A}(\mathbf{q}) \ddot{\mathbf{q}}+\mathbf{C}(\mathbf{q}, \dot{\mathbf{q}}) \dot{\mathbf{q}}+\mathbf{G}(\mathbf{q})+\Gamma_{s}=\mathbf{B} \Gamma
$$


where $\mathbf{A}(q) \in \mathbb{R}^{6 \times 6}$ is the positive definitive inertia matrix, $\mathbf{C}(q, \dot{q}) \in \mathbb{R}^{6 \times 6}$ contains the Coriolis and centrifugal forces, $\mathbf{G}(q) \in \mathbb{R}^{6 \times 1}$ is the vector of gravity forces, $\mathbf{B} \in \mathbb{R}^{6 \times 6}$ is the actuation matrix consisting of zeros and ones and $\boldsymbol{\Gamma}_{s} \in \mathbb{R}^{6 \times 1}$ is the vector of spring torque.

\subsection{Optimization Criterion}

In optimization problem, reference trajectory for a walking step is generated and then optimized to minimize a criterion shown in (3) under constraints. We are using a polynomial of order four for the evolution of actuated joints motion in function of time to generate reference trajectory.

$$
C_{\Gamma}=\frac{1}{d} \int_{0}^{T} \Gamma^{\mathrm{t}} \boldsymbol{\Gamma} d t
$$

The above criterion was optimized using MATLAB function fmincon under inequality constraints of max. joint torques, max. joint velocities, max. Joint angles, Zero Moment Point (ZMP) and no slipping and take-off of support foot during swing phase.

\section{Results}

Our results show that the most effective way to reduce energy consumption during walking is to add springs to the support joints only and the most economical is the support knee joint (see fig. 2) which economizes up to $92 \%$ of energy at $0.30 \mathrm{~m} / \mathrm{sec}$.

Adding springs to the support leg only is possible by having a variable spring stiffness mechanism ${ }^{11}$ capable of producing spring stiffness from zero to required value, however this mechanism will add an additional mass and the robot's parameters will change. In our simulation tests, we assumed that this mechanism is massless and the energy consumed by the mechanism is negligible. The time of activation and deactivation of the variable spring is very important. If the spring is not activated at the right time, energy is lost. To avoid this variable stiffness mechanism, we restrained ourselves to the simple option and studied the effects of adding identical torsional springs to both legs.

Figure 1 presents the value of objective function as a function of walking speed for our biped in case $\mathbf{A}$, case $\mathbf{B} \mathbf{1}$ where spring was introduced to the support leg and case $\mathbf{B 2}$ where identical springs were added to both of the legs. It is to be noted that identical springs were added to the same joint 


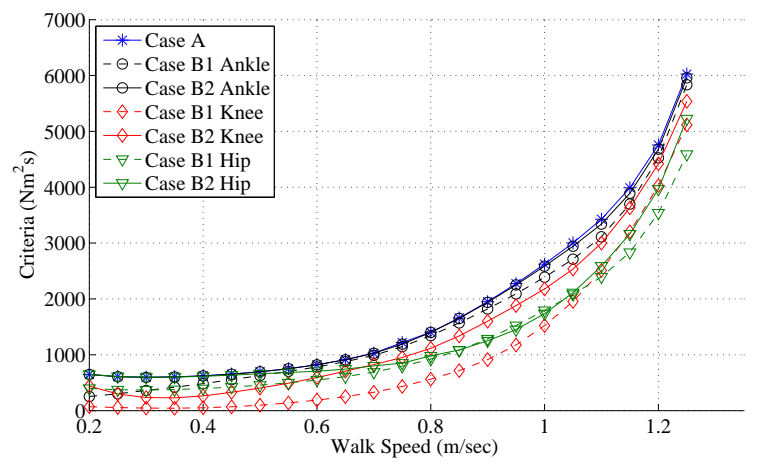

Fig. 1. Value of objective function as a function of walk speed (solid lines for springs at both legs and dashed lines for springs at support leg)

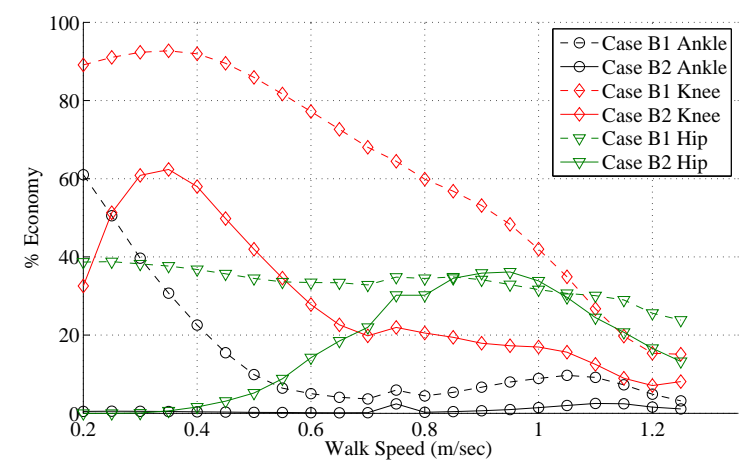

Fig. 2. Percentage energy savings as a function of walk speed (solid lines for springs at both legs and dashed lines for springs at support leg)

of both legs. Figure 1 shows that the optimization criteria is significantly reduced after introducing identical springs to both knee or hip joints in parallel with existing actuator.

Figure 2 case $\mathbf{B 2}$ indicates that knee springs are effective at low walking speeds while hip springs are effective for hight walking speeds. The two curves cross at walking speed of $0.70 \mathrm{~m} / \mathrm{sec}$ which means that if the application area is below this point then use knee springs otherwise use hip springs. It was however observed that adding springs to both ankles were not effective for our robot. This is contrary to Ref. 7 and Ref. 10 where they found ankle springs useful for stability and energetic efficiency. This is because we considered a flat foot impactless walking gait and there is no 
rotation of support foot during entire swing phase and in addition we kept the spring offset angle zero which can improve the role of ankle springs.

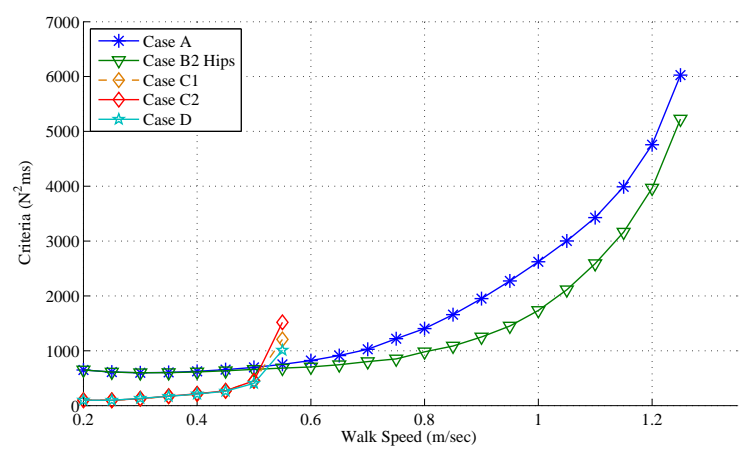

Fig. 3. Value of objective function as a function of walk speed

Figure 3 gives comparison of criteria curves as a function of walking speed in case $\mathbf{A}$, case $\mathbf{B 2}$ (identical springs on both hips), case $\mathbf{C} 1$ where knee locking angle $\beta$ was optimized, case $\mathbf{C 2}$ where $\beta$ of 8.3 degree was obtained from optimization results in case $\mathbf{C} \mathbf{1}$ and for a biped in case $\mathbf{D}$ with an identical spring on both hips and support knee locked. Hip springs are selected here for comparison and eventual addition along with knee locking because it is not possible to study spring effects on the knee joint while the joint is locked.

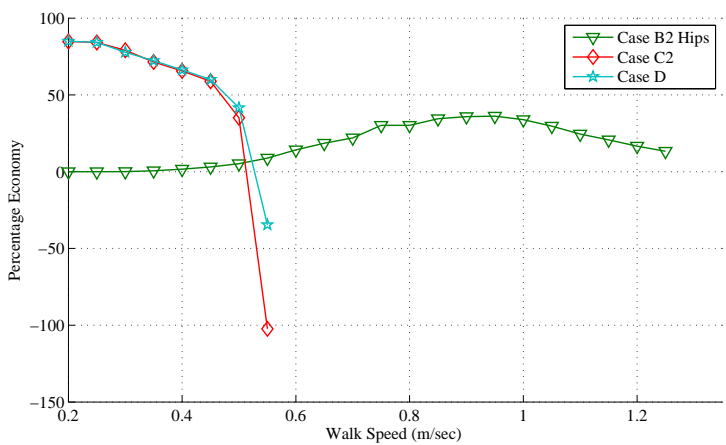

Fig. 4. Percentage energy savings as a function of walk speed 
Our simulation results show that knee locking for an impactless bipedal walking gait is economical at lower walking speeds but more costly at higher speeds. The two curves in case $\mathbf{C} \mathbf{1}$ and $\mathbf{C 2}$ are superposed which clearly indicate that knee can be locked at a constant angle for all possible walking speeds. It is also evident from figure 3 that addition of springs at hip joints while support knee is locked has negligible effects on energy saving compared to knee locked only.

It was also observed that no feasible walk trajectory was found above $0.6 \mathrm{~m} / \mathrm{sec}$ with support knee locked while without knee locking, the biped was able to walk at $1.25 \mathrm{~m} / \mathrm{sec}$. When the support knee was locked, the ZMP constraint was not satisfied at walking speeds above $0.6 \mathrm{~m} / \mathrm{sec}$.

Figure 4 shows that the energetic efficiency in case $\mathbf{D}$ is almost the same as that of in case $\mathbf{C 2}$. It is clear from our simulation results that knee locking is effective at lower walking speeds (upto $0.5 \mathrm{~m} / \mathrm{sec}$ ) and for speeds above $0.5 \mathrm{~m} / \mathrm{sec}$; the only choice is the hip springs.

\section{Conclusion and Perspectives}

In this paper impactless trajectories were generated for a seven-link bipedal robot with three different strategies, one by adding torsional springs to different joints, second by mechanically locking the knee and third by combining the two strategies i.e. locking the support knee and adding identical springs to hip joints. The key focus of the study was to economize the energy consumption during walking.

We conclude from this study that the energy consumption of a biped was significantly reduced by adding identical torsional springs at the hip joint and mechanically locking the knee however the maximum attainable walking speed was reduced by a small amount. The energetic efficiency of blocking the knee alone at low speeds and adding springs only to the knee or hip joints is also noticeable. Practical implementation of this strategy will significantly improve the energetic efficiency as well as the autonomy of our biped.

In line with the previous research, our study reinforces the idea of using passive joint stiffness to improve energetic efficiency of the biped especially on the hip joints in our case. However, contrary to previous work, ankle springs were not effective in our study.

In perspective of this study, the next step is to explore the effects of spring offset or bias angle and the ankle springs with foot rotation on the energetic efficiency of the biped. This can also be extended to study effects on a $3 \mathrm{D}$ bipedal robot. 


\section{Acknowledgment}

This work is supported by ANR grants for the R2A2 project and by Higher Education Commission (HEC) of Pakistan under overseas scholarship program.

\section{References}

1. S. Alfayad, F.B. Ouezdou, and F. Namoun. New three dof ankle mechanism for humanoid robotic application: Modeling, design and realization. In Proceedings of the IEEE/RSJ International Conference on Intelligent Robots and Systems (IROS 2009), pages 4969-4976, 2009.

2. S.H. Collins and A. Ruina. A bipedal walking robot with efficient and human-like gait. In Proceedings of the 2005 IEEE International Conference on Robotics and Automation (ICRA 2005), pages 1983-1988, 2005.

3. Heinz Ulbrich Daniela Frg, Martin Frg. A bipedal robot model with elastic actuation. In Proceedings of the 1st Joint International Conference on Multibody System Dynamics, May 25-27 2010.

4. Arthur D. Kuo. Energetics of actively powered locomotion using the simplest walking model. Journal of Biomechanical Engineering, 124(1):113-120, 2002.

5. Cara L. Lewis and Daniel P. Ferris. Walking with increased ankle pushoff decreases hip muscle moments. Journal of Biomechanics, 41(10):2082-2089, 2008.

6. Xiuhua Ni, Weishan Chen, and Junkao Liu. A comparison between human walking and passive dynamic walking. In Proceedings of the 4 th IEEE Conference on Industrial Electronics and Applications (ICIEA 2009), pages 2552$2555,2009$.

7. T. Schauss, M. Scheint, M. Sobotka, W. Seiberl, and M. Buss. Effects of compliant ankles on bipedal locomotion. In Proceedings of the IEEE International Conference on Robotics and Automation (ICRA '09), pages 2761-2766, 2009.

8. K. Trifonov and S. Hashimoto. Active knee-lock release for passive-dynamic walking machines. In Proceedings of the IEEE International Conference on Robotics and Biomimetics (ROBIO 2007), pages 958-963, 2007.

9. Gijs Oort van, Raffaella Carloni, Dian J. Borgerink, and Stefano Stramigioli. An energy efficient knee locking mechanism for a dynamically walking robot. In IEEE International Conference on Robotics and Automation, ICRA 2011, USA, May 2011. IEEE Robotics and Automation Society.

10. M. Wisse, D.G.E. Hobbelen, R.J.J. Rotteveel, S.O. Anderson, and G.J. Zeglin. Ankle springs instead of arc-shaped feet for passive dynamic walkers. In Proceedings of the 6th IEEE-RAS International Conference on Humanoid Robots, pages 110-116, 2006.

11. J. Yamaguchi, D. Nishino, and A. Takanishi. Realization of dynamic biped walking varying joint stiffness using antagonistic driven joints. In Proceedings of the IEEE 1998 IEEE International Conference on Robotics and Automation, volume 3, pages 2022-2029 vol.3, 1998. 